\title{
Lapatinib plus Capecitabine for HER2-Positive Advanced-Stage Breast Cancer in Elderly Women: Review of the Anatolian Society of Medical Oncology (ASMO) Experience
}

\author{
Bulent Cetin $^{a} \quad$ Mustafa Benekli $^{\mathrm{a}}$ Faysal Dane $^{\mathrm{b}} \quad$ Cem Boruban $^{\mathrm{c}}$ Mahmut Gumus $^{\mathrm{d}}$ \\ Berna Oksuzoglue Mehmet A. Kaplan ${ }^{f}$ Gulnihal Tufan ${ }^{a}$ Alper Sevinc ${ }^{g}$ \\ Ugur Coskun $^{\mathrm{a}}$ Suleyman Buyukberber ${ }^{\mathrm{a}}$
}

aDepartment of Medical Oncology, Gazi University Faculty of Medicine, Ankara, ${ }^{\mathrm{b} D e p a r t m e n t ~ o f ~ M e d i c a l ~ O n c o l o g y, ~ M a r m a r a ~ U n i v e r s i t y ~}$ Faculty of Medicine, Istanbul, 'Department of Medical Oncology, Selcuk University Meram Faculty of Medicine, Konya, ${ }^{\mathrm{d} D e p a r t m e n t}$ of Medical Oncology, Dr. Lutfi Kirdar Kartal Training and Research Hospital, Istanbul, e'Department of Medical Oncology, Dr. Abdurrahman Yurtaslan Training and Research Hospital, Ankara, 'Department of Medical Oncology, Dicle University Faculty of Medicine, Diyarbakir, ${ }^{9}$ Department of Medical Oncology, Gaziantep University Faculty of Medicine, Gaziantep, Turkey

\section{Keywords}

Advanced breast cancer - Elderly women - Lapatinib . Capecitabine $\cdot$ HER2

\section{Summary}

Background: The efficacy and safety of the lapatinib and capecitabine combination remain elusive in elderly patients with metastatic breast cancer $(\mathrm{MBC})$, who progress after trastuzumab-based therapy. Patients and Methods: A total of 26 patients with HER2-positive MBC were included in this retrospective multicenter study. Median age was 69 years (range 65-82 years). All patients were treated with the combination of lapatinib $(1,250 \mathrm{mg} / \mathrm{day}$, continuously $)$ and capecitabine $\left(2,000 \mathrm{mg} / \mathrm{m}^{2}\right.$ on days $1-14$ of a 21 -day cycle $)$. Data on demographics, clinical outcome, and toxicity were collected for descriptive analyses. Results: The median follow-up was 10 months (range 2-31 months). An overall response rate of $33.4 \%$ was achieved, including 1 complete response $(3.8 \%)$, and 8 partial responses $(30.8 \%)$. Median progression-free survival was 7 months $(95 \%$ confidence interval (Cl) 5-8), and the median overall survival was 15 months (95\% Cl 11-19). Most common side effects were fatigue $(53.8 \%)$, diarrhea (46\%), vomiting $(36.3 \%)$, hand-foot syndrome $(34.5 \%)$, and anorexia (34.6\%). Grade 3-4 toxicities were identified as hand-foot syndrome (3.8\%), diarrhea $(7.6 \%)$, and fatigue $(11.5 \%)$. There were no symptomatic cardiac events. Conclusion: Lapatinib and capecitabine combination therapy was effective and well tolerated in elderly patients with MBC, who had progressive disease after trastuzumab-based therapy.

\section{Schlüsselwörter \\ Fortgeschrittenes Mammakarzinom - Ältere Frauen · Lapatinib · Capecitabine · HER2}

\section{Zusammenfassung}

Hintergrund: Die Effizienz und Sicherheit der Kombination aus Lapatinib und Capecitabin bei älteren Patientinnen mit metastasiertem Mammakarzinom und Krankheitfortschreiten nach Trastuzumab-Therapie ist noch immer ungeklärt. Patientinnen und Methoden: Insgesamt wurden 26 Patientinnen mit HER2-positivem metastasierten Mammakarzinom in diese retrospektive multizentrische Studie aufgenommen. Das mittlere Alter war 69 Jahre (Spanne 65-82 Jahre). Alle Patientinnen wurden mit einer Kombination aus Lapatinib (1250 mg/d, kontinuierlich) und Capecitabine $(2000 \mathrm{mg} /$ $\mathrm{m}^{2}$ Tag 1-14 eines 21-tägigen Zyklus) behandelt. Demographische Daten sowie Daten zu klinischem Outcome und Toxizität wurden zur Durchführung beschreibender Analysen gesammelt. Ergebnisse: Das mittlere Follow-Up war 10 Monate (Spanne 2-31 Monate). Eine Gesamtansprechrate von $33,4 \%$ wurde erzielt, einschließlich eines Falls (3,8\%) mit komplettem Ansprechen und 8 Fällen (30,8\%) mit teilweisem Ansprechen. Das mittlere progressionsfreie Überleben war 7 Monate (95\%-Konfidenzinterval (KI) 5-8), und das mittlere Gesamtüberleben war 15 Monate (95\%-KI 11-19). Die häufigsten Nebenwirkungen waren Fatigue (53,8\%), Durchfall (46\%), Erbrechen (36,3\%), Hand-Fuß-Syndrom (34,5\%) und Appetitlosigkeit (34,6\%). Als Toxizitäten vom Grad 3-4 traten das Hand-Fuß-Syndrom (3,8\%), Durchfall $(7,6 \%)$ und Fatigue $(11,5 \%)$ auf. Symptomatische kardiale Nebenwirkungen wurden nicht beobachtet. Schlussfolgerung: Die Kombination aus Lapatinib und Capecitabin war bei älteren Patientinnen mit metastasiertem Mammakarzinom und Krankheitfortschreiten nach Trastuzumab-Therapie effektiv und wurde gut toleriert. 


\section{Introduction}

Approximately $15-20 \%$ of all breast cancers are human epidermal growth factor receptor 2 (HER2)-positive which has historically been associated with poorer disease-free and overall survival (OS) [1]. The incidence of breast cancer is 2-3 times higher in women aged $\geq 65$ years than in the whole population, whereas their mortality rate is 3- to 4 -fold higher [2]. Although the rate of HER2 positivity is $20-25 \%$ overall, it is lower in older patients. Older women with breast cancer are underrepresented in clinical trials, and data on effects of targeted therapy especially when combined with chemotherapy in such patients are scant.

Metastatic breast cancer (MBC) is generally not curable with current treatment modalities. Management focuses on palliation and symptom control, control of tumor growth, and prolongation of progression-free survival (PFS) and OS. Given the palliative nature of such treatments, extreme care must be taken to adequately assess each individual's potential for both benefit and harm from chemotherapy.

Lapatinib is a small molecule tyrosine kinase inhibitor that targets both HER2 and the epidermal growth factor receptor 1 (EGFR1; ErbB1; HER1). Several studies demonstrated the efficacy and safety of lapatinib either as monotherapy or in combination with chemotherapy or endocrine therapy in patients with MBC [3-7]. Lapatinib exhibits a good overall tolerance, but no study has yet been dedicated to elderly women. Lapatinib is approved for the treatment of advanced breast cancer or MBC in patients not responding to trastuzumab, combined with capecatabine chemotherapy. The present study was designed to evaluate the efficacy and tolerability of the combination of lapatinib and capecitabine in patients with $\mathrm{MBC}$, who were 65 years of age or older and progressed after trastuzumab-based therapy.

\section{Patients and Methods}

\section{Study Population}

The medical records of elderly patients ( $\geq 65$ years) with HER2-positive MBC, who had received lapatinib and capecitabine combination after progression following trastuzumab and an anthracycline and/or taxane chemotherapy, were retrospectively analyzed at 7 centers between September 2007 and May 2011. All patients had received prior trastuzumab-based therapies in the adjuvant setting or for metastatic disease. Eligible patients had a median age of 69 years (range 65-82 years) and HER2-positive, locally advanced (stage IIIB or IIIC with T4 lesion) or metastatic breast cancer (stage IV) that had progressed (as assessed by modified RECIST) following treatment with an anthracycline and/or a taxane and trastuzumab alone or in combination in either the adjuvant or the metastatic setting. HER2 positivity was determined locally and defined as immunohistochemical staining of $3+$ or $2+$ with evidence of gene amplification determined by fluorescence in situ hybridization. Patients received lapatinib $1,250 \mathrm{mg}$ once daily at approximately the same time every morning and capecitabine $2,000 \mathrm{mg} / \mathrm{m}^{2} /$ day, divided into 2 doses, days 1-14, every 21 days. Dose reductions and delays for lapatinib- and/or capecitabine-related toxic effects were permitted.
Data on demographics, clinical outcome, and toxicity were collected based on retrospective evaluation of medical records for the descriptive analyses.

\section{Evaluation of Efficacy}

PFS was the time elapsed from the date of initiation of lapatinib plus capecitabine to the date of first evidence of disease progression or death in the absence of disease progression. OS was defined as the period from the first day of treatment until the date of last follow-up or death. Disease progression was assessed using the modified Response Evaluation Criteria in Solid Tumors (RECIST) version 1.1. Patient follow-up generally consisted of regular physical examination and laboratory assessment (hematologic and serum chemical measurements), every 4-6 weeks, left ventricular ejection fraction (LVEF) evaluation and computed tomography (CT) scans were performed according to the local standard every 12-18 weeks. Patients with partial remission (PR) and complete remission (CR) were considered as responders.

Table 1. Demographic and baseline clinical characteristics in patients with HER2+ breast cancer treated with lapatinib plus capecitabine $(\mathrm{n}=26)$

\begin{tabular}{lc}
\hline Age, median (range), years & $69(65-82)$ \\
ECOG performance status, n (\%) & \\
0 & $6(23.1)$ \\
1 & $13(50.0)$ \\
2 & $7(26.9)$ \\
Hormone receptor status, n (\%) & \\
ER- and/or PR-positive & $14(53.8)$ \\
ER- and PR-negative & $12(46.1)$ \\
Metastatic sites, n (\%) & \\
Liver & $12(46.1)$ \\
Bone & $19(73.0)$ \\
Lung & $13(50.0)$ \\
Brain & $7(26.9)$ \\
Other (local, axilla, skin) & $9(34.6)$ \\
Prior chemotherapy, n (\%) & \\
(Neo)-adjuvant & $15(57.8)$ \\
Metastatic 1 & $0(0.0)$ \\
Metastatic 2 & $6(23.1)$ \\
Metastatic 3 & $10(38.5)$ \\
Metastatic > 3 & $10(38.4)$ \\
Previous therapy, n (\%) & $16(61.5)$ \\
Anthracyclines & $24(92.3)$ \\
Taxanes & $4(15.4)$ \\
Capecitabine & $8(30.7)$ \\
Vinorelbine & $5(19.2)$ \\
Gemcitabine & $15(57.6)$ \\
Type of prior transtuzumab therapy, n (\%) & $11(42.3)$ \\
Adjuvant only & $26(100.0)$ \\
Metastatic & $36(8-152)$ \\
Both adjuvant and metastatic & \\
Prior trastuzumab, median (range), weeks & \\
\hline ER = Estrogen receptor; PR = progesterone receptor. \\
(n)
\end{tabular}

$\mathrm{ER}=$ Estrogen receptor; $\mathrm{PR}=$ progesterone receptor.

Table 2. Efficacy data in patients with HER2+ breast cancer treated with lapatinib plus capecitabine $(\mathrm{n}=26)$

\begin{tabular}{lc}
\hline Overall survival, median (range), months & $15(11-19)$ \\
Progression-free survival, median (range), months) & $7(5-8)$ \\
Overall response, \% & 34.2 \\
Complete response, n (\%) & $1(3.8)$ \\
Partial response, n (\%) & $8(30.8)$ \\
Stable disease, n (\%) & $10(38.5)$ \\
Progressive disease, n (\%) & $7(26.9)$ \\
Clinical benefit, n (\%) & $19(72.7)$ \\
\hline
\end{tabular}


Table 3. Adverse events in patients with HER2+ breast cancer treated with lapatinib plus capecitabine $(\mathrm{n}=26)$

\begin{tabular}{lcl}
\hline Adverse event & Any grade, $\mathrm{n}(\%)$ & Grade 3-4, $\mathrm{n}(\%)$ \\
\hline Hand-foot syndrome & $9(34.5)$ & $1(3.8)$ \\
Diarrhea & $12(46)$ & $2(7.6)$ \\
Fatigue & $14(53.8)$ & $3(11.5)$ \\
Nausea & $4(15.3)$ & $0(0.0)$ \\
Rash & $4(15.4)$ & $0(0.0)$ \\
Anorexia & $9(34.6)$ & $1(3.8)$ \\
Vomiting & $9(34.6)$ & $0(0.0)$ \\
Stomatitis & $6(23.1)$ & $0(0.0)$ \\
Abdominal pain & $2(7.6)$ & $0(0.0)$ \\
Dyspnea & $1(3.8)$ & $0(0.0)$ \\
Constipation & $1(3.8)$ & $0(0.0)$ \\
\hline
\end{tabular}

\section{Evaluation of Safety}

Patients were monitored for serious adverse events (SAEs). Adverse events (AEs) were assessed according to the National Cancer Institute's Common Terminology Criteria for Adverse Events (CTCAE, version 3.0) which grades events as mild (grade 1), moderate (grade 2), severe (grade 3), life-threatening or disabling (grade 4), or fatal (grade 5). Concerning cardiac safety, a cardiac event was defined as a decline in the LVEF that was symptomatic regardless of the degree of decline, or was asymptomatic but with a relative decrease of $20 \%$ or more from baseline to a level below the institution's lower limit of the normal range. Lapatinib was discontinued in patients with symptomatic cardiac events.

\section{Statistical Analysis}

Statistical analysis was made using computer software (SPSS version 17.0, SPSS Inc. Chicago, IL, USA). Descriptive statistics were determined for patient demographics and clinical characteristics. Kaplan-Meier curves were used to summarize PFS and OS. Data were expressed as 'mean (standard deviation; SD)', minimum-maximum (range), percent (\%) and $95 \%$ confidence interval $(\mathrm{CI})$, where appropriate. $\mathrm{p}<0.05$ was considered statistically significant.

\section{Results}

Patient demographics and baseline clinical characteristics are summarized in table 1 . Mean age was 69 years (range 65-82 years), and 19 patients $(73.1 \%)$ had a performance status score ranging from 0 to 1 . The median duration of previous treatment with trastuzumab was determined to be 36 weeks. The most common non-central nervous system (CNS) metastatic sites were liver (46.1\%), bone (73\%) and lung (50\%), with brain metastases in $26.9 \%$ of the patients. Patients with brain metastases were evaluated separately elsewhere [8]. The patients had previously received anthracyclines and taxanes either in the (neo)-adjuvant setting or for metastatic disease (antracyclines, $\mathrm{n}=16$; taxanes, $\mathrm{n}=24$ ).

\section{Efficacy Results}

A total of 194 treatment cycles were administered. Among all 26 patients, there were 1 (3.4\%) with CR, 8 (30.8\%) with PR $10(38.5 \%)$ with stable disease (SD), and $7(26.9 \%)$ with progressive disease (PD), resulting in an overall response rate (ORR) of $34.2 \%$. The clinical benefit rate (ORR plus SD) was $72.7 \%(\mathrm{n}=19)($ table 2$)$. In the OS analysis, 12 patients

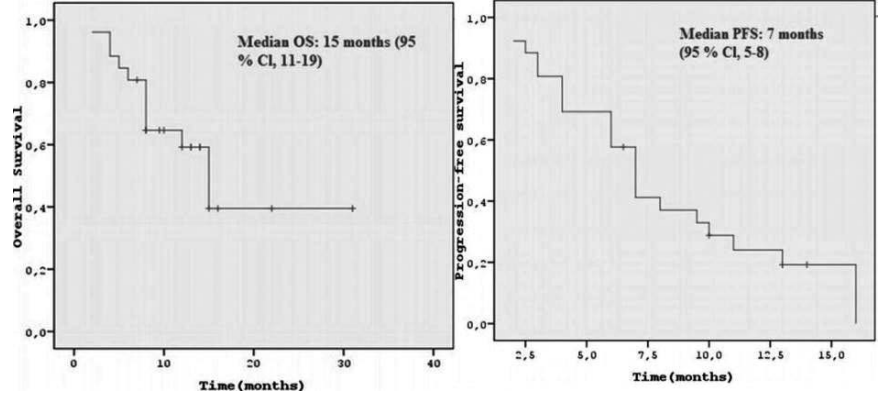

Fig. 1. Overall survival (OS): median OS was 16 months (95\% CI 11-19). Progression-free survival (PFS): median PFS was 7 months (95\% CI 5-8).

had died (46.1\%). The median OS was 15 months (95\% CI 11-19) (fig. 1). The median PFS was 7 months (95\% CI 5-8).

\section{Adverse Events}

The combination therapy was generally well tolerated. The most common AEs were diarrhea, hand-foot syndrome, vomiting, fatigue, and anorexia (table 3 ). In 1 patient treatment was permanently discontinued due to grade 4 neutropenia and grade 4 diarrhea. There was no episode of febrile neutropenia. In addition, 1 patient with grade 3 hand-foot syndrome had interrupted the combination therapy for up to 14 days. After recovery from grade 3 toxicity lapatinib was to be resumed at a dose of $1,000 \mathrm{mg} / \mathrm{day}$, and capecatabine was to be resumed at dose of $1,500 \mathrm{mg} / \mathrm{m}^{2}$ daily on days $1-14$ of a 21-day cycle. Concerning cardiac safety, 1 patient in the lapatinib and capecitabine combination treatment was noted to have experienced a cardiac side effect as evidenced by a reduction of $10 \%$ in LVEF, and lapatinib was withheld; however symptoms had resolved on follow-up assessment, and lapatinib was resumed at a dose of $1,000 \mathrm{mg} /$ day.

\section{Discussion}

EGFR-targeted therapies have created a new environment for the development of clinical trials and disease management in patients with MBC. One of the major caveats of clinical trials in oncology relates to the limited information that we have in the elderly population, a particularly common group of patients with this disease $[9,10]$. Whether or not tumors in the elderly are less aggressive than those occurring in young women remains uncertain. To our knowledge, this report is the first to address the efficacy and tolerability of the combination of lapatinib and capecitabine therapy in an elderly breast cancer population. According to our findings concerning retrospective evaluation of the efficacy and tolerability of lapatinib and capecitabine in HER2-positive MBC patients who had progressed after trastuzumab-based therapy, revealed the combination treatment to be effective and well tolerated with a median PFS of 7 months, an ORR of $34.2 \%$, and a median OS of 15 months. 
In the Lapatinib Expanded Access Program (LEAP), median PFS and OS were reported to be 21.1 (95\% CI 20.1-22.3) and 39.6 (95\% CI 37.7-40.7) weeks, respectively [11]. In another study (EGF100151), median time to progression as assessed by the independent review committee (time from randomization to disease progression or death from breast cancer) was documented as 27.1 weeks (6.2 months), and median OS was 67.7 weeks (15.6 months) [5]. There is a lack of data from studies specifically designed for the elderly populations, which would be able to address the question of efficacy and tolerability of therapeutic choices. Therefore, recommendations are not based on level I evidence. The most important overall limits to inclusion of elderly patients in clinical trials are the reluctance of investigators and the inclusion and exclusion criteria usually defined by the investigative team of the trial $[2,9]$.

In the current study, the tolerance profile of lapatinib and capecitabine combination in elderly women was good; the main AEs were concerning the gastrointestinal system (diarrhea) and dermatologic symptoms. The most frequently reported events were hand-foot syndrome, diarrhea, fatigue, anorexia, and vomiting. Moreover, the most common grade 3 or 4 AEs were diarrhea and fatigue. Approximately $38.1 \%$ of the reported SAEs in LEAP were possibly related to lapatinib, and the most frequently reported events were diarrhea, vomiting, and nausea. In EGF100151, the most common AEs (> 25\% incidence, any grade) reported for patients receiving lapatinib plus capecitabine were diarrhea, nausea, vomiting, hand-foot syndrome, and rash. Moreover, the most common grade 3 or 4 AEs in EGF100151 were diarrhea and hand-foot syndrome [4]. Cardiotoxicity was rare, although impairment of the LEVF was documented [11]. Combination therapy of lapatinib and capecitabine was neither associated with an increase in the number of SAEs nor with the prevalence of discontinuation of treatment due to these side effects. There were no withdrawals from treatment due to declines in LVEF, no cases of congestive heart failure, and no decreases in the mean LVEF values under lapatinib and capecitabine combination treatment.

We recognize the limitations of this study. First, the older patients in this analysis represent a highly selected population referred to tertiary care cancer centers. Therefore, this study may not be representative of the general population of elderly patients with advanced breast cancer, limiting the ability to generalize the results. Second, it is important to remember that all patients were deemed suitable candidates for lapatinib and capecatabine combination therapy in our study. It is likely that older patients with a poorer performance status would not have been considered for chemotherapy and would instead have been prescribed hormonal therapy or no treatment at all. Our study has shown that lapatinib-based therapy is well tolerated in elderly MBC patients. However prospective clinical trials including the elderly population may help to elucidate the efficacy and toxicity of these novel targeted therapies and to avoid both underuse and overtreatment.

\section{Disclosure Statement}

The authors declare no conflict of interests.

\section{References}

1 Slamon DJ, Clark GM, Wong SG, Levin WJ, Ullrich A, McGuire WL: Human breast cancer: correlation of relapse and survival with amplification of the HER-2/neu oncogene. Science 1987; 235:177-182.

2 Wildiers H, Kunkler I, Biganzoli L, et al.: Management of breast cancer in elderly individuals: recommendations of the International Society of Geriatric Oncology. Lancet Oncol 2007;8:1101-1115.

>3 Johnston S, Pippen J Jr, Pivot X, et al.: Lapatinib combined with letrozole versus letrozole and placebo as first-line therapy for postmenopausal hormone receptor-positive metastatic breast cancer. J Clin Oncol 2009;27:5538-5546.

$\checkmark 4$ Geyer CE, Forster J, Lindquist D, et al.: Lapatinib plus capecitabine for HER2-positive advanced breast cancer. N Engl J Med 2006;355:2733-2743.
5 Cameron D, Casey M, Press M, et al.: A phase III randomized comparison of lapatinib plus capecitabine versus capecitabine alone in women with advanced breast cancer that has progressed on trastuzumab: updated efficacy and biomarker analyses. Breast Cancer Res Treat 2008;112:533-543.

6 Di Leo A, Gomez HL, Aziz Z, et al.: Phase III, double-blind, randomized study comparing lapatinib plus paclitaxel with placebo plus paclitaxel as first-line treatment for metastatic breast cancer. J Clin Oncol 2008;26:5544-5552.

7 Gomez HL, Doval DC, Chavez MA, et al.: Efficacy and safety of lapatinib as first-line therapy for Her2-amplified locally advanced or metastatic breast cancer. J Clin Oncol 2008;26:2999-3005.

8 Cetin B, Benekli M, Oksuzoglu B, et al.: Lapatinib plus capecitabine for brain metastases in patients with human epidermal growth factor receptor 2-positive advanced breast cancer: a review of Anatolian Society of Medical Oncology (ASMO) experience. Onkologie 2012;35:740-745.

9 Hutchins LF, Unger JM, Crowley JJ, Coltman CA Jr, Albain KS: Underrepresentation of patients 65 years of age or older in cancer-treatment trials. N Engl J Med 1999;341:2061-2067.

10 Yancik R, Wesley MN, Ries LA, Havlik RJ, Edwards BK, Yates JW: Effect of age and comorbidity in postmenopausal breast cancer patients aged 55 years and older. JAWA 2001;285:885-892.

11 Capri G, Chang J, Chen SC, et al.: An open-label expanded access study of lapatinib and capecitabine in patients with HER2-overexpressing locally advanced or metastatic breast cancer. Ann Oncol 2010;21:474-480. 